\title{
Design and implementation of time synchronization system based on GPS signal
}

\author{
Liu bin, Shuling Guo, Shasha Ma \\ 1Northwest Industrial Group Co.,Ltd.,Happiness South Road Lane \\ 2Northwest Industrial Group Co.,Ltd.,Happiness South Road Lane \\ 3Xi'an, Shaanxi Province, 710043, China
}

Keywords: GPS signal; time synchronization; time calibration.

\begin{abstract}
The GPS synchronization system can provide accurate time reference to system testing. To improve the accuracy and stability of the synchronous timer in GPS, this paper presents a new method to implement highly accurate and stable clock by the synchronization of crystal oscillator clock with GPS clock, which is based on the complement of the characteristics between GPS and crystal oscillator. And it can ensure the system performing normal testing by local time running under the circumstance of invalid GPS signal.
\end{abstract}

\section{Introduction}

The high accurate time reference of Global Positioning System (GPS) has become one of the basic guarantee platforms for industrial control, communications, radio and television and other fields. For its excellent timing performance, all-weather and all-time positioning and navigation services, GPS is widely used in many areas, such as computers on the internet, CDMA base station etc. which need to coordinate and synchronize with local needs. Capitalizing on the excellent features of GPS, the time synchronization device realized through the double research and development has several advantages which traditional timing mode was incomparable [1]. In this paper, using the excellent characteristics and the periphery interface features of GPS OEM timing mode, the author has designed a completely automatic time synchronization system, which was composed of hardware interface circuit and corresponding software.

\section{GPS timing principle}

A new type of timing applications product GPS OEM, formed on the newest high accuracy GPS positioning module, can simultaneously receive 4 11 satellites signals within the range of its vision, and output timing and positioning information format conforming to the regulation, thus to complete time service. Under normal GPS signal circumstances, there will be outputting two kinds of timing signals:

i ) 1PPS signals with a second interval. Its synchronization error between its pulse front edge and UTC absolute time outputted from serial port does not exceed 1us.

ii ) Signals included in the RS232 serial output information in the UTC (Coordinate Universal Time) absolute time, corresponded with second pulse, as it is shown in the figur1.

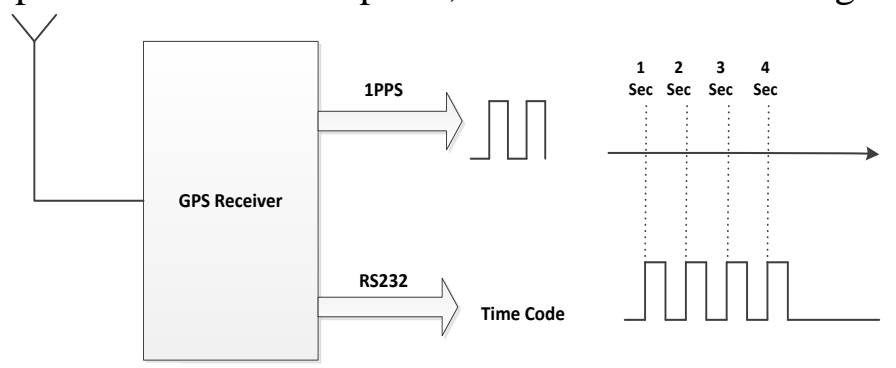

Fig.1: Relationship between the second pulse and UTC standard time data of GPS 
The GPS receiver outputs1PPS signal with 1 second interval, which corresponds to a rising edge of the accurate UTC time [2]. Therefore, the rising edge can be used to trigger and control other equipment, thus to achieve the accurate time when second pulse comes, and complete time synchronization work. However, it is difficult to receive satellite signals when the GPS service module used in the places Such as the room, tunnel and shelter or electromagnetic interference environment, in which signal coverage is weak. During this time, timing signal will appear short disorder or unstable situation [3]. So, in order to ensure the test precision of the system, it is necessary to design a perfect timekeeping program.

\section{Design of GPS time synchronization system}

\section{The time calibration method of GPS synchronization clock}

GPS timing module itself provides the very accurate time information, but in which way to calibrate the system has become the key issue to ensure the accuracy of time synchronization. The timing method of GPS synchronous clock mainly has the following three:

i ) Time calibration of serial communication

Time calibration of serial communication refers to that serial port outputs time information by the way of string data stream, and every base station receives time information with fixed update rate when calibrating. In this process, interrupting mode was used to send and receive serial data. Therefore, the time of CPU will be occupied by the interrupt handler of both parties, and the length of time is related to the interrupt priority settings of both serial ports. In addition, data byte is transmitted bit-by-bit by certain baud rate. So the bus transmission will also have a certain time delay, which can be accurately calculated, mainly related to the amount of data transmission and the serial baud rate. Whereas other factor affecting the time calibration such as interrupt processing delay can only be made at a rough estimate. Therefore, the above factors should be taken into consideration to make sure the precision of time calibration when using serial communication, thus to make a compensation though a given modified value.

ii ) Time calibration of pulse interruption

Time calibration of pulse interrupt refers to the GPS timing module is used to output a precise synchronous pulse signal at a certain interval, in which the system was calibrated to remove its accumulated errors of local clock when CPU has received the pulse. During this way, although the signal transmission, isolation and response and processing of interrupt program will be delayed, the whole delay time is about tens of microseconds [4]. So the accuracy can meet the demands of the system, even if the time correction was not made.

iii) Comprehensive time calibration

When being considered of the calibration mode of serial communication and pulse interrupt, if only the former was used, the accuracy is far from the demands of testing system because of the time of data transmitted and dealt in the bus will accumulate to millisecond level. Therefore, it's vital to make time calibration. And the size of correction value must depend on the specific situation to the scene, which brings great inconveniences to users. While if only the latter was used, it is comparatively easier but corresponding time and positioning information were not acquired and system were unable to effectively test. Thus, these two ways can be properly combined to offer accurate and reliable timekeeping service for the system.

\section{The design of complete timekeeping system}

GPS receiver could output high accuracy, safe and reliable timing signal under the normal GPS signal. But in some extreme circumstances such as continuous rainy days and places where signal coverage is weaker, the GPS signal may not be well received by antenna. Therefore, the condition of invalid GPS must be considered to ensure the timing accuracy of output signal. When the GPS receiver emerges transient asynchrony or unstable output, the timing accuracy of synchronous system mainly depends on local clock, from which reference signal was generated for travel time. The Oven Controlled Crystal Oscillator (OCXO) belongs to precision temperature control device, from which crystal was always made to work near the zero-temperature coefficient point through constant 
temperature devices. The OCXO has very high frequency accuracy and stability, whose performance is almost close to the level of atomic clock. It performs well in aging rate, temperature stability etc. and even better than the atomic clock in the short-term stability. So, the OCXO was used in this paper to achieve local timekeeping function. The system diagram has been shown in the figure2.

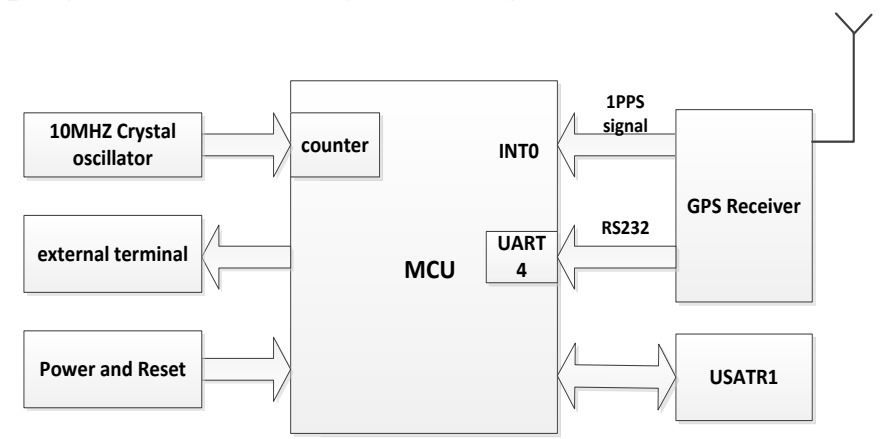

Fig.2: Principle diagram of time synchronization system

In order to ensure that the time synchronization of testing system was at the same time level, the control port should firstly be initialized when system was powered. Before test was started, valid GPS_1PPS second pulse signal should be received at least once, during which the counting processing is still continued. Local clock was refreshed from zero when the GPS_1PPS second pulse signal is valid, in the same meaning of resetting the local second counter, and signal generated at next second will be synchronized with GPS_1PPS second pulse signal.

\section{Design and implementation of time synchronization system}

\section{The design and realization of hardware}

i ) Design of GPS receiving module circuit

The high performance LEA-6T time module of Switzerland U-blox was selected as the GPS_OEM board, which the first positioning time is less than 1 second. In the static operation, accurate time could be output even if the GPS signal is worse and sky visibility in poor circumstances, and also remove the timing error. Its precision can be as high as $15 \mathrm{~ns}$ by using the quantization error to compensate the error of time pulse particles. A variety of data outputted from GPS_OEM serial port were received by system CPU, and the UTC data was distracted by designing corresponding software, which was also converted into Beijing time. In addition, CPU is also responsible for receiving the second pulse from GPS_OEM and performs local travel time by internal counter, as is shown in the figure3.

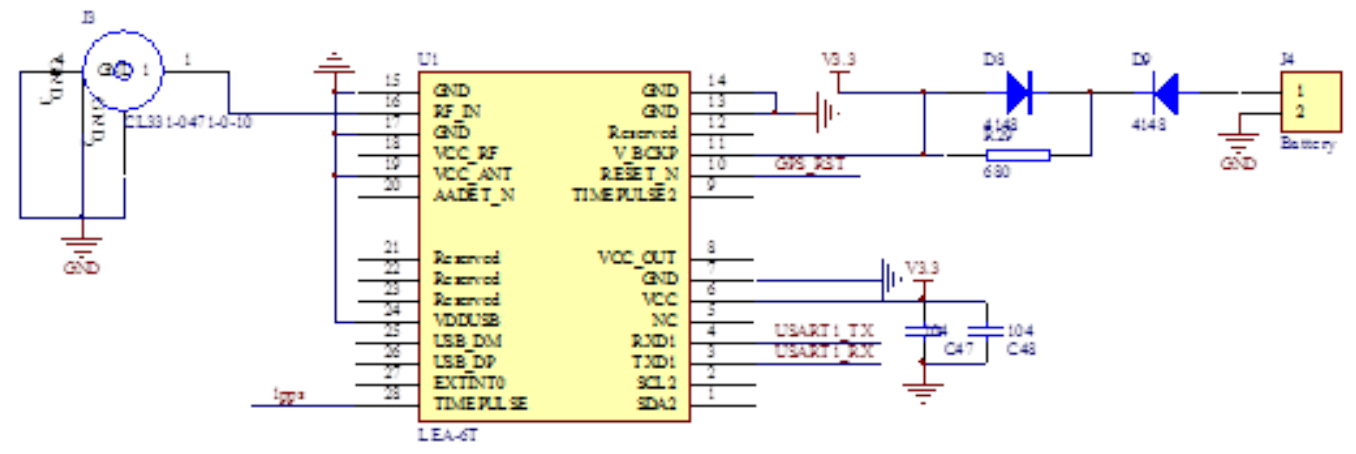

Fig.3: Working circuit of GPS_OEM

LEA-6T serial communication baud rate can be selected by setting 4800bps, 9600bps on the CFG_COM1 port. TIMEPULSE and TIMEPULSE2 pin can provide with 1PPS second pulse signal corresponding to UTC time, in which the pulse rising edge is corresponded to positive second moment. The UART port supports 7messages'(GSV, RMC, GSA, GGA, GLL, VTG, TXT) transmission of NMEA-0183 protocol, of which the VCC power is 3.3V. To improve the stability of power, two $0.1 \mathrm{uF}$ bypass capacitors were paralleled. The standby voltage is $3.3 \mathrm{~V}$. The UART charges 
and discharges the battery by two diodes D8、D9. And UART1 is responsible for uploading time information or downloading initialization procedure for GPS.

ii ) Design of clock circuit

The basic function of the clock circuit is to keep track of time information, which instead of GPS travels time in case GPS is out of step. It requires better clock accuracy in the whole system, because the time error resulted from timing rate of clock will have impacts on the results of time synchronization, while the errors of ordinary passive in-line crystal is greater due to the influence of temperature, humidity, air pressure and other factors. So OCXO constant temperature crystal oscillator of $10 \mathrm{MHZ}$ has been used in this design, of which the accuracy is $0.1 \mathrm{ppm}$ and daily timekeeping stability is only tens of milliseconds. The clock circuit is shown in the figure4.

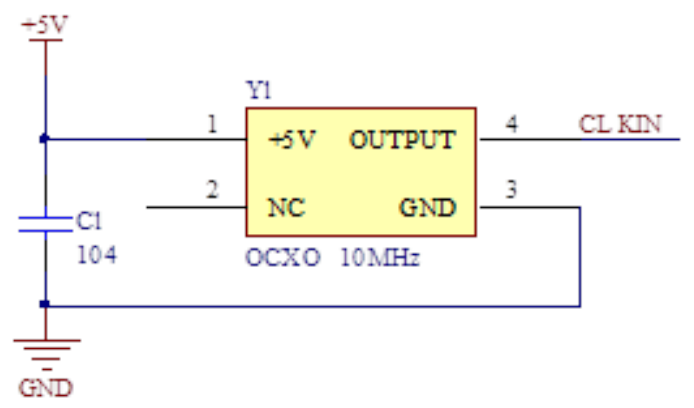

Fig.4: Clock circuit

\section{The design and realization of software}

i ) Extraction of GPS module information

GPS timing module will continuously send received satellites signals after which were processed to processor as long as it is in the working condition. Because suffering from several errors like ionosphere error, troposphere error, satellite clock error, multipath error and few number of tracking satellite, GPS often cannot enter the location condition. Therefore, it is extracting the valid GPS information to judge that became the effective and reliable measures to determine the GPS information.

7 messages of NMEA-0183 protocol outputted from GPS, of which GPS positioning condition, time, date and other fundamental factors are offered, while current number of visible satellites and status information of various satellites are contained in the \$GPGSV. The criterion for judgment of the reliability of GPS information is that it can be considered current outputting GPS is valid, when met the demands of positioning state information and several satellites tracked, otherwise it will be considered invalid.

8 hours were added to the UTC (Universal Time Coordinated) from GPS and then it is converted to Beijing time. GPS message is extracted by comma as a separator, in which the two characters after the third comma of \$GPGSV message indicate the number of satellites. GPS positioning state is extracted by comma as a separator, in which the first character after the second comma. A represents successful positioning and V the failed position. Extraction of GPS is as follows: Time information is described as the first to sixth character after the first comma of \$GPRMC message, while date information is described as the first to sixth character after the ninth comma of \$GPRMC message.

ii ) The counter module

Counting part uses internal timer or counter of processor as the constitution of local timing suit. Counting pulse can be obtained by the local $10 \mathrm{MHz}$ oscillator frequency. The cycle is 1us when counting pulse is 10 dividing frequency of system clock signal. Internal 16 bit timer or counter of MCU implements the clock by counting the $1 \mathrm{MHZ}$ signal, which is divided into real time counter and microsecond counter. When the second pulse does work, the local counter is reset by GPS' second pulse and restarted, and the synchronization between local clock and standard GPS second pulse is implemented the next moment. When the GPS signal is out of step, the local 1PPS second pulse can be used as a short time standard second time signal to realize local travel time. 


\section{Test of time synchronization performance}

In order to test the time precision of time synchronous device, any two of GPS device were placed in the open area, and was connected with an external interrupt button through peripheral hardware circuit. The time information of interrupt arrival will be recorded respectively by GPS time synchronization device when the button is pressed. And this will be delivered to the PC port through data transmission module. Repeat the above steps in the room.

By testing, the time error is within 5us when GPS signal is normal. But it will down to 0.1ms when the GPS signal is invalid after 2hours, thus to ensure the system stably and accurately output in a long time.

Grid1 Testing data of GPS time synchronization device

Table 1: Testing data of GPS time synchronization device

\begin{tabular}{cccc}
\hline Serial number & Device I trigger time & Device II trigger time & Relative error \\
\hline 1 & $10: 16: 12$ & $10: 16: 12$ & $1 \mu \mathrm{s}$ \\
(GPSnormal) & $779 \mathrm{~ms} 359 \mu \mathrm{s}$ & $779 \mathrm{~ms} 360 \mu \mathrm{s}$ & $2 \mu \mathrm{s}$ \\
\hline 2 & $10: 21: 46$ & $10: 21: 46$ & \\
(GPS normal) & $560 \mathrm{~ms} 117 \mu \mathrm{s}$ & $560 \mathrm{~ms} 119 \mu \mathrm{s}$ & $4 \mu \mathrm{s}$ \\
3 & $11: 25: 09$ & $11: 25: 09$ & \\
(GPS normal) & $676 \mathrm{~ms} 413 \mu \mathrm{s}$ & $676 \mathrm{~ms} 417 \mu \mathrm{s}$ & $2 \mu \mathrm{s}$ \\
4 & $11: 26: 11$ & $11: 26: 11$ & \\
(GPS normal) & $224 \mathrm{~ms} 699 \mu \mathrm{s}$ & $224 \mathrm{~ms} 701 \mu \mathrm{s}$ & $26 \mu \mathrm{s}$ \\
5 & $13: 31: 05$ & $13: 31: 05$ & \\
(GPS invalid ) & $452 \mathrm{~ms} 812 \mu \mathrm{s}$ & $452 \mathrm{~ms} 838 \mu \mathrm{s}$ & $31 \mu \mathrm{s}$ \\
6 & $13: 32: 52$ & $13: 32: 52$ & \\
\hline
\end{tabular}

\section{Conclusions}

Aiming at the high accuracy and great reliability of testing system, this paper, utilizing the complementary characteristics between GPS time module and OCXO, has designed a new type of timekeeping device with good stability and high time precision, of which the time error is within $0.1 \mathrm{~ms}$. With the development of a number of large scale electronic information systems, it has a great significance to keep exploring ways of working on GPS timing signal.

\section{References}

[1]Chen Junyong. Toward the twenty-first century Global Positioning System and its Application[J].Journal of Surveying and mapping,1999, 28(1):6 10.

[2]Zhang Chao, Zheng Yong. Research of Accurate Timing by Using GPS OEM Chip[J].Journal of Information Engineering University, 2001, 2(4):50 53.

[3]Yang Yongbiao, Yang Xiaoyu and Zhou Jie. Realize Accurate Timing with FPGA during GPS syn Failure[J].Electric Power Automation Equipment, 2007,27(7):109 112.

[4]Zhang Shouxin. Theory and application of GPS satellites positioning measurement [M].Changsha: National University of Defense Technology press, 1996.

[5]YUAN Hai-bo. The Study on the Automatic Steering Method of UTC(NTSC) and the Design of Its Software[D]. Graduate University of Chinese Academy of Sciences(NTSC),2005.

[6]He Hong-bing. The Research and Design of High Precision Time Synchronization System Based on GPS[D]. Sichuan University,2005. 
[7]Cadenas,O.;Megson,G.Pipelining Considerations for an FPGA case[A].Proceedings of IEEE International Symposuim on Digital Systems Design[C],2001,276-285.

[8]A Technical Tutorial on Digital Signal Synthesis[M].Analog Device Inc,USA,1999:45-58.

[9]David Finkleman,Steve Allen.The Future of Time:UTC and the Leap Second[J].American Scientist,2011,99(4).

[10]Moore P,Crossley P.GPS Applictions in Power System:I.introduction to GPS[J].Power Engineering Jounral,2011,99(4). 